\title{
Betweenness centrality in convex amalgamation of graphs*
}

Research Article

\section{Sunil Kumar Raghavan Unnithan, Kannan Balakrishnan}

\begin{abstract}
Betweenness centrality measures the potential or power of a node to control the communication over the network under the assumption that information flows primarily over the shortest paths between pair of nodes. The removal of a node with highest betweenness from the network will most disrupt communications between other nodes because it lies on the largest number of paths. A large network can be thought of as inter-connection between smaller networks by means of different graph operations. Thus the structure of a composite graph can be studied by analysing its component graphs. In this paper we present the betweenness centrality of some classes of composite graphs constructed by the graph operation called amalgamation or merging.
\end{abstract}

2010 MSC: 05C75, 05C76, 05C 85

Keywords: Betweenness centrality, Central vertex, Extreme vertex, Convex subgraph, Vertex amalgamation, Edge amalgamation, Path amalgamation, Subgraph amalgamation.

\section{Introduction}

A large network can be thought of as inter-connection between smaller networks by means of different graph operations. Graph operations are important for constructing new classes of composite graphs and many of the structural properties of larger graphs can be derived from their component graphs. There are many operations on two graphs $G_{1}$ and $G_{2}$ which result in a larger graph $G$.

In this paper we define some betweenness centrality concepts, and derive the betweenness centrality for some classes of composite graphs constructed by the graph operation subgraph-amalgamation.

* This work was supported by the University Grants Commission (UGC), Government of India under the scheme of Faculty Development Programme (FDP) for colleges.

Sunil Kumar Raghavan Unnithan (Corresponding Author), Kannan Balakrishnan; Department of Computer Applications, Cochin University of Science and Technology, Kerala, India (email: sunilstands@gmail.com, mullayilkannan@gmail.com). 


\section{Some betweenness centrality concepts}

The concept of betweenness centrality of a vertex was first introduced by Bavelas in 1948 [1].

Definition 2.1. [3]. Let $G$ be a graph and $x \in V(G)$, then the betweenness centrality of $x$ in $G$, denoted by $B_{G}(x)$ or simply $B(x)$ is defined as

$$
B_{G}(x)=\sum_{s, t \in V(G) \backslash\{x\}} \frac{\sigma_{s t}(x)}{\sigma_{s t}}
$$

where $\sigma_{s t}(x)$ denotes the number of shortest $s$ - $t$ paths in $G$ passing through $x$ and $\sigma_{\text {st }}$, the total number of shortest $s$ - $t$ paths in $G$. The ratio $\frac{\sigma_{s t}(x)}{\sigma_{s t}}$ is called pair dependency or partial betweenness of $(s, t)$ on $x$, denoted by $\delta_{G}(s, t, x)$.

Betweenness centrality of some well known graphs has been studied in [7] and we use the following definitions [8].

\subsection{Betweenness centrality of a vertex in a subgraph}

Definition 2.2. Let $G$ be a graph and $H$ a subgraph of $G$. Let $x \in V(H)$, then the betweenness centrality of $x$ in $H$ denoted by $B_{H}(x)$ is defined as

$$
B_{H}(x)=\sum_{s, t \in V(H) \backslash\{x\}} \frac{\sigma_{s t}^{H}(x)}{\sigma_{s t}^{H}}
$$

where $\sigma_{s t}^{H}(x)$ and $\sigma_{s t}^{H}$ denotes the number of shortest $s$-t paths passing through $x$ and the total number of shortest $s$-t paths respectively, lying in $H$.

\subsection{Betweenness centrality of a vertex induced by a subgraph}

Definition 2.3. Let $G$ be a graph and $H$ a subgraph of $G$. Let $x \in V(G)$, then the betweenness centrality of $x$ induced by $H$ denoted by $B(x, H)$ is defined as

$$
B(x, H)=\sum_{s, t(\neq x) \in V(H)} \frac{\sigma_{s t}(x)}{\sigma_{s t}}
$$

where $\sigma_{s t}(x)$ and $\sigma_{\text {st }}$ denotes the number of shortest $s$-t paths passing through $x$ and the total number of shortest $s$-t paths respectively in $G$.

The betweenness centrality of a vertex induced by a subset $S \subset V(G)$ is defined likewise.

\subsection{Betweenness centrality of a vertex induced by a subset}

Definition 2.4. Let $G$ be a graph and $S$ a subset of $V(G)$. Let $x \in V(G)$, then the betweenness centrality of $x$ induced by $S$ denoted by $B(x, S)$ is defined as

$$
B(x, S)=\sum_{s, t(\neq x) \in S} \frac{\sigma_{s t}(x)}{\sigma_{s t}}
$$

where $\sigma_{s t}(x)$ and $\sigma_{\text {st }}$ denotes the number of shortest $s$-t paths passing through $x$ and the total number of shortest $s$-t paths respectively in $G$. 


\subsection{Betweenness centrality of a vertex induced by another vertex}

Definition 2.5. Let $G$ be a graph and $s, x, t \in V(G)$, then the betweenness centrality of $x$ induced by $s$ in $G$, denoted by $B_{G}(x, s)$ or simply $B(x, s)$ is defined by

$$
B_{G}(x, s)=\sum_{t \in V(G) \backslash x} \frac{\sigma_{s t}(x)}{\sigma_{s t}} .
$$

It can be easily seen that in any graph $G$, the betweenness centrality induced by a vertex on its extreme vertex or an end vertex is zero. Consider the following examples.

$$
B\left(x_{i}, x_{j}\right)=0 \text { for } x_{i}, x_{j} \in K_{n} \text {. Let } P_{n} \text { be a path on } n \text { vertices }\left\{x_{1}, \ldots, x_{n}\right\} \text {, then }
$$

$$
B\left(x_{i}, x_{j}\right)= \begin{cases}i-1, & \text { if } i<j, \\ n-i, & \text { if } j<i .\end{cases}
$$

If $C_{n}$ is a cycle on $n$ vertices $\left\{x_{0}, \ldots, x_{n-1}\right\}$, then if $n$ is even,

$$
B\left(x_{i}, x_{0}\right)= \begin{cases}\frac{n-1-2 i}{2}, & \text { if } 1 \leq d\left(x_{i}, x_{0}\right)<n / 2 \\ 0, & \text { if } d\left(x_{i}, x_{0}\right)=n / 2\end{cases}
$$

if $n$ is odd,

$$
B\left(x_{i}, x_{0}\right)=\frac{n-1-2 i}{2}, \quad \text { if } 1 \leq d\left(x_{i}, x_{0}\right) \leq \frac{n-1}{2}
$$

For a star $S_{n}$ with central vertex $x_{0}$,

$$
\begin{gathered}
B\left(x_{i}, x_{0}\right)=0, B\left(x_{0}, x_{i}\right)=n-2 \text { and } \\
B\left(x_{i}, x_{j}\right)=0 \text { for } i, j \neq 0 .
\end{gathered}
$$

For a wheel $W_{n}, n>5$ with central vertex $x_{0}$,

$$
B\left(x_{i}, x_{0}\right)=0, B\left(x_{0}, x_{i}\right)=n-5 .
$$

For $i, j \neq 0$,

$$
B\left(x_{i}, x_{j}\right)= \begin{cases}1 / 2, & \text { if } d\left(x_{i}, x_{j}\right)=1 \\ 0, & \text { if } d\left(x_{i}, x_{j}\right)=2\end{cases}
$$

It can be easily seen that for $x_{i} \in V(G), B_{G}\left(x_{i}\right)=\frac{1}{2} \sum_{j \neq i} B_{G}\left(x_{i}, x_{j}\right)$.

\subsection{Betweenness centrality donated by a vertex}

Definition 2.6. Let $G$ be a graph and $x_{0} \in V(G)$, then the betweenness centrality donated by $x_{0}$ in $G$, denoted by $D B_{G}\left(x_{0}\right)$ or simply $D B\left(x_{0}\right)$, is defined as the sum of betweenness values induced by $x_{0}$ on all other vertices in $G$, i.e.,

$$
D B_{G}\left(x_{0}\right)=\sum_{x \in V(G) \backslash x_{0}} B_{G}\left(x, x_{0}\right) .
$$

The betweenness centrality received by a vertex, $R B_{G}\left(x_{0}\right)$ is $B_{G}\left(x_{0}\right)$ by definition. 


\subsection{Betweenness centrality of a vertex induced by two disjoint subsets}

Definition 2.7. Let $G$ be a graph and $x \in V(G)$. Let $S, T$ be two disjoint subsets of $V(G)$, then the betweenness centrality of $x$ induced by $S$ and $T$ denoted by $B(x, S, T)$ is defined as

$$
B(x, S, T)=B(x, S)+B(x, T) .
$$

2.7. Betweenness centrality of a vertex induced by two disjoint subsets, one against the other

Definition 2.8. Let $G$ be a graph and $x \in V(G)$. Let $S, T$ be two disjoint subsets of $V(G)$ where $s(\neq x) \in S$ and $t(\neq x) \in T$, then the betweenness centrality of $x$ induced by $S$ against $T$, denoted by $B(x, S \mid T)$ is defined as

$$
B(x, S \mid T)=\sum_{s \in S, t \in T} \frac{\sigma_{s t}(x)}{\sigma_{s t}}
$$

where $\sigma_{s t}(x)$ and $\sigma_{\text {st }}$ denotes the number of shortest $s$ - $t$ paths passing through $x$ and the total number of shortest $s$ - $t$ paths respectively in $G$.

In metric graph theory, a convex subgraph of an undirected graph $G$ is a subgraph that includes every shortest path in $G$ between two of its vertices. A subgraph $H$ of a graph $G$ is an isometric subgraph, if $d_{H}(u, v)=d_{G}(u, v)$ for all $u, v \in V(H)$. Clearly, a convex subgraph is an isometric subgraph, but the converse need not be true.

\section{Subgraph-amalgamation}

One method of constructing composite graphs is merging or pasting two or more graphs together along a common subgraph. For any finite collection of graphs $G_{i}$, each with a fixed isomorphic subgraph $H$ as common, the subgraph-amalgamation is the graph obtained by taking the union of all the $G_{i}$ and identifying their fixed subgraphs $H$ 's. The simplest one is vertex-amalgamation or vertex-merging.

Theorem 3.1. Let $G$ be the graph obtained by merging the graphs $\left\{G_{i}\right\}_{i=1}^{n}$ along $n$ copies of isomorphic induced common convex subgraph $H$ where $H \subset G_{i} \forall i$. Let $S_{i}=V\left(G_{i}-H\right) \forall i$. Then, for $x \in H$ and $u \in G_{k}-H$

$$
\begin{aligned}
& B_{G}(x)=\sum_{i=1}^{n} B_{G_{i}}(x)-(n-1) B_{H}(x)+\sum_{i<j} B\left(x, S_{i} \mid S_{j}\right), \\
& B_{G}(u)=B_{G_{k}}(u)+\sum_{i \neq k} B\left(u, S_{i} \mid S_{k}\right) .
\end{aligned}
$$

Proof. Consider the graphs $\left\{G_{i}\right\}_{i=1}^{n}$. Let $G$ be the graph obtained by merging $G_{i}$ along $n$ copies of isomorphic induced common convex subgraph $H$ where $H \subset G_{i} \forall i$. See Figure 1 . Now the subgraphs $G_{i}-H$ and $H$ form a partition of $G$. Any path joining a vertex of $G_{i}-H$ and a vertex of $G_{j}-H$ for $i \neq j$ passes through at least one vertex of $H$. Let $x \in H$, then its betweenness centrality $B_{G}(x)$ in $G$ is due to the contribution of possible pairs of vertices from each $G_{i}$ and from different pairs of $G_{i}-H$. Since $H$ is convex, $B_{H}(x)$ is repeated $n$ times in $\sum_{i=1}^{n} B_{G_{i}}(x)$ and hence we get $B_{G}(x)=$ $\sum_{i=1}^{n} B_{G_{i}}(x)-(n-1) B_{H}(x)+\sum_{i<j} B\left(x, S_{i} \mid S_{j}\right)$. Let $u \in G_{k}-H$. To find $B_{G}(u)$, consider a pair of vertices $s, t \in G$ which contributes to $B_{G}(u)$, then $s \in S_{k}$, and $t$ may be either in $G_{k}$ or in its complement $G_{k}^{\prime}$. Those $t \in G_{K}$ gives $B_{G_{k}}(u)$ and those $t \in G_{k}^{\prime}$ gives $B\left(u, S_{i} \mid S_{k}\right)$ for $i \neq k$. Hence $B_{G}(u)=B_{G_{k}}(u)+\sum_{i \neq k} B\left(u, S_{i} \mid S_{k}\right)$. 


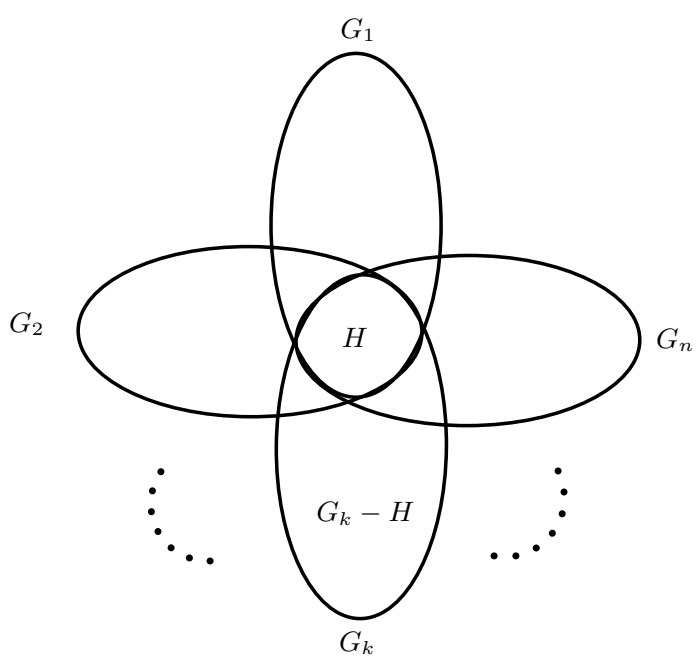

Figure 1. Subgraph-amalgamation

Algorithm 3.2. Algorithm for Betweenness Computation of a Vertex $x \in V(H)$ in Subgraph Amalgamation

Require: Graphs $G_{1}, G_{2}, \ldots, G_{n}$, convex common subgraph $H$, vertex $x \in H$

Ensure: Betweenness of vertex $x, B_{G}(x)$

1: $S_{i}=V\left(G_{i}-H\right)$ for $i=1, \ldots, n$.

2: Compute betweenness of $x$ in $G_{i}, B_{G_{i}}(x)$ for $i=1, \ldots, n$. Add all these betweenness values to get $B_{\text {ComponentSum }}(x)$

3: Compute betweenness of $x$ in $H, B_{H}(x)$.

4: Compute betweenness of $x$ in $S_{i}, B_{S_{i}}(x)$ for $i=1, \ldots, n$.

5: Find $B\left(x, S_{i} \mid S_{j}\right)=\sum_{s \in S_{i}, t \in S_{j}} \frac{\sigma_{s t}(x)}{\sigma_{s t}}$ for all $i<j, i=1, \ldots, n$. Add all these betweenness values to get $B_{\text {SubsetSum }}(x)$

6: Determine $B_{G}(x)=B_{\text {ComponentSum }}(x)-(n-1) * B_{H}(x)+B_{\text {SubsetSum }}(x)$

Theorem 3.3. Let $G$ be the graph obtained by merging the graphs $\left\{G_{i}\right\}_{i=1}^{n}$ along $n$ copies of isomorphic induced common convex subgraph $H$ where $H \subset G_{i} \forall i$. Then Algorithm 3.2 correctly computes the betweenness value of vertex $x \in H$ in $O\left(n^{\prime} m^{\prime}\right)$ time where $n^{\prime}$ is the order of the largest component graph $G_{i}$ and $m^{\prime}$ the number of edges in $G_{i}$.

Proof. Proof of Algorithm 3.2 follows from Theorem 3.1.

The efficient algorithm by Brandes [2] compute betweenness centrality of all vertices in an unweighted graph in $O(n m)$ time and $O(m+n)$ space where $m$ is the number of edges in the graph and $n$ is the number of vertices. Here we compute the betweenness centrality of a vertex using partial betweenness of different components of the amalgamation. Therefore, the complexity gets reduced to $O\left(n^{\prime} m^{\prime}\right)$ where $n^{\prime}$ is the order of the largest component graph $G_{i}$ and $m^{\prime}$ the number of edges in $G_{i}$.

Algorithm 3.4. Algorithm for Betweenness Computation of Vertex $u$ in $G_{k}-H$ in a Subgraph Amalgamation

Require: Graphs $G_{1}, G_{2}, \ldots, G_{n}$, convex common subgraph $H$, vertex $u \in V\left(G_{k}\right)-V(H)$

Ensure: Betweenness of vertex $x, B_{G}(x)$

1: $S_{i}=V\left(G_{i}-H\right)$ for $i=1, \ldots, n$

2: Compute betweenness of $u$ in $G_{k}, B_{G_{k}}(u)$

3: Compute betweenness of $u$ in $S_{i}, B_{S_{i}}(u)$ for $i=1, \ldots, n$. 
4: Find $B\left(u, S_{i} \mid S_{k}\right)=\sum_{s \in S_{i}, t \in S_{k}} \frac{\sigma_{s t}(u)}{\sigma_{s t}}$ for all $i \neq k$. Add all these betweenness values to get $B_{\text {SubsetSum }}(u)$

5: Determine $B_{G}(u)=B_{G_{k}}(u)+B_{\text {SubsetSum }}(x)$

Theorem 3.5. Let $G$ be the graph obtained by merging the graphs $\left\{G_{i}\right\}_{i=1}^{n}$ along $n$ copies of isomorphic induced common convex subgraph $H$ where $H \subset G_{i} \forall i$. Then Algorithm 3.4 correctly computes the betweenness value of vertex $u$ in $G_{k}-H$ in $O\left(n_{k} m_{k}\right)$ time where $n_{k}$ is the order of $G_{k}$ and $m_{k}$ the number of edges in $G_{k}$.

Proof. Proof of Algorithm 3.4 follows from Theorem 3.1.

\subsection{Path-amalgamation of graphs}

\section{Two cycles connected by merging along a common subgraph}

Proposition 3.6. Let $G$ be the graph obtained by merging two cycles $C_{m}$ and $C_{n}$ along a common path $P_{p}=\left\{x_{1}, \ldots, x_{p}\right\}$ as common subgraph where $p<\min \left\{\left\lceil\frac{m}{2}\right\rceil,\left\lceil\frac{n}{2}\right\rceil\right\}$ and $U=\left\{u_{1}, \ldots, u_{m-p}\right\}$ and $V=\left\{v_{1}, \ldots, v_{n-p}\right\}$, be the vertex sets of $C_{m}-P_{p}$ and $C_{n}-P_{p}$ respectively, then the betweenness centrality of $C_{m}$ in $G$ is given by

$$
\begin{aligned}
& B_{G}\left(x_{r}\right)=B_{C_{m}}\left(x_{r}\right)+B_{C_{n}}\left(x_{r}\right)-(r-1)(p-r)+B\left(x_{r}, U \mid V\right), \text { for } x_{r} \in P_{p}, \\
& B_{G}\left(u_{r}\right)=B_{C_{m}}\left(u_{r}\right)+B\left(u_{r}, U \mid V\right), \text { for } u_{r} \in U,
\end{aligned}
$$

where $B\left(x_{r}, U \mid V\right)$ and $B\left(u_{r}, U \mid V\right)$ are given by

Case 1: When $C_{m}$ and $C_{n}$ are even

$$
\begin{aligned}
& B\left(x_{r}, U \mid V\right)= \begin{cases}m n / 2-(m+n)(p-1 / 2)+2\left(p^{2}-p+1 / 3\right), & \text { for } 1<r<p, \\
3 m n / 4-(m+n)(p-1 / 4)+\left(9 p^{2}-6 p+2\right) / 6, & \text { for } r=1, p .\end{cases} \\
& B\left(u_{r}, U \mid V\right)= \begin{cases}(n-p)(m-p-2 r) / 2, & \text { for } 1 \leq r \leq \frac{m}{2}-p, \\
n(2 p-3) / 4-\left(3 p^{2}-3 p-2\right) / 6, & \text { for } r=\frac{m}{2}-p+1, \\
k(k-p+1)+(n-p)(p-2) / 2+1 / 6, & \text { for } r=\frac{m}{2}-p+1+k, 1 \leq k \leq p-2 .\end{cases}
\end{aligned}
$$

Case 2: When $C_{m}$ and $C_{n}$ are odd

$$
\begin{aligned}
& B\left(x_{r}, U \mid V\right)= \begin{cases}2[(m+1) / 2-p][(n+1) / 2-p], & \text { for } 1<r<p, \\
3 m n / 4-(m+n)(p-1 / 4)+\left(6 p^{2}-4 p+1\right) / 4, & \text { for } r=1, p .\end{cases} \\
& B\left(u_{r}, U \mid V\right)= \begin{cases}(n-p)(m-p-2 r) / 2, & \text { for } 1 \leq r \leq \frac{m+1}{2}-p, \\
(p-2)(n-p-1) / 2, & \text { for } r=\frac{m+1}{2}-p+1, \\
k(k-p+2)+(p-2)(n-p-1) / 2, & \text { for } r=\frac{m+1}{2}-p+1+k, 1 \leq k \leq p-2 .\end{cases}
\end{aligned}
$$

Case 3: When $C_{m}$ is even and $C_{n}$ is odd

$$
\begin{gathered}
B\left(x_{r}, U \mid V\right)= \begin{cases}(m-2 p+1)(n-2 p+1) / 2, & \text { for } 1<r<p, \\
3 m n / 4-(m+n)(p-1 / 4)+\left(6 p^{2}-4 p+1\right) / 4, & \text { for } r=1, p .\end{cases} \\
B\left(u_{r}, U \mid V\right)= \begin{cases}(n-p)(m-p-2 r) / 2, & \text { for } 1 \leq r \leq \frac{m}{2}-p, \\
1 / 2[(n-p)(p-2)+(n+1) / 2-p], & \text { for } r=\frac{m}{2}-p+1, \\
k(k-p+1)+(n-p)(p-2) / 2, & \text { for } r=\frac{m}{2}-p+1+k, 1 \leq k \leq p-2 .\end{cases} \\
B\left(v_{r}, U \mid V\right)= \begin{cases}(m-p)(n-p-2 r) / 2, & \text { for } 1 \leq r \leq \frac{n+1}{2}-p, \\
1 / 2\left[m(p-2)-p^{2}+p+2\right], & \text { for } r=\frac{n+1}{2}-p+1, \\
k(k-p+2)+(p / 2)(m-p+1)-m+1, & \text { for } r=\frac{n+1}{2}-p+1+k, 1 \leq k \leq p-2 .\end{cases}
\end{gathered}
$$


Proof. Let $C_{m}$ and $C_{n}$ be two cycles merged along a common path $P_{p}$ induced by the common vertices $X=\left\{x_{1}, \ldots, x_{p}\right\}$ where $p<\min \left\{\left\lceil\frac{m}{2}\right\rceil,\left\lceil\frac{n}{2}\right\rceil\right\}$. Let $U=\left\{u_{1}, \ldots, u_{m-p}\right\}$ and $V=\left\{v_{1}, \ldots, v_{n-p}\right\}$ be the remaining vertices in $C_{m}$ and $C_{n}$ respectively. Consider the three cycles $C_{m}, C_{n}$ and $C^{\prime}$, where $C^{\prime}$ is the cycle induced by $U, V,\left\{x_{1}\right\}$ and $\left\{x_{p}\right\}$. By symmetry, the vertices in each of the sets $X, U, V$ from either ends have the same betweenness centrality in $G$.

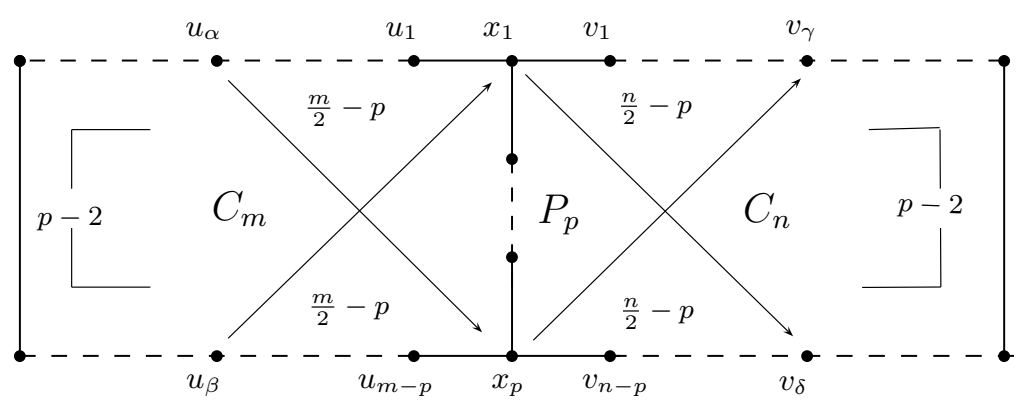

Figure 2. Two even cycles merged along a common subgraph

Case 1: Both $C_{m}$ and $C_{n}$ are even.

Let $u_{\alpha}, u_{\beta}$ be the eccentric vertices of $x_{p}$ and $x_{1}$ respectively in $C_{m}$ and $v_{\delta}, v_{\gamma}$ be their eccentric vertices in $C^{\prime}$ where $\alpha=\frac{m}{2}-p+1, \beta=\frac{m}{2}, \gamma=\frac{n}{2}-p+1$ and $\delta=\frac{n}{2}$. See Figure 2. Let $x_{r} \in X$. By Theorem 3.1, $B_{G}\left(x_{r}\right) \stackrel{2}{=} B_{C_{m}}\left(x_{r}\right)+B_{C_{n}}\left(x_{r}\right)-B_{P}\left(x_{r}\right)+B\left(x_{r}, U \mid V\right)$. Let us find $B\left(x_{r}, U \mid V\right)$ as the others are known. Consider $u \in U$ and $v \in V$, then for each $(u, v)$ pair there are at most three shortest $u-v$ paths and in the case of $\left(u_{\alpha}, v_{\delta}\right)$ and $\left(u_{\beta}, v_{\gamma}\right)$ there are three shortest $u-v$ paths $P_{1}$, $P_{2}$ and $P_{3}$ passing through the end vertices $x_{1}, x_{p}$ and the whole path $P$.

(a) Consider the vertex $x_{r}$ for $1<r<p$, then those pairs $\left(u_{i}, v_{j}\right)$ for $1 \leq i \leq \alpha, \delta \leq j \leq n-p$, or $\left(u_{i}, v_{j}\right)$ for $\beta \leq i \leq m-p, 1 \leq j \leq \gamma$ contribute betweenness centrality $\left(\frac{m}{2}-p\right)\left(\frac{n}{2}-p\right)+\frac{1}{2}\left(\frac{m}{2}-\right.$ $\left.p+\frac{n}{2}-p\right)+\frac{1}{3}$ to $x_{r}$. Thus by Theorem 3.1 we get,

$B_{G}\left(x_{r}\right)=\frac{1}{8}\left[(m-2)^{2}+(n-2)^{2}\right]-(r-1)(p-r)+2\left[\left(\frac{m}{2}-p\right)\left(\frac{n}{2}-p\right)+\frac{1}{2}\left(\frac{m}{2}-p+\frac{n}{2}-p\right)+\frac{1}{3}\right]$.

(b) Consider the vertex $x_{1}$, then the pairs $\left(u_{i}, v_{j}\right)$ for $1 \leq i<\alpha, 1 \leq j \leq n-p$ contributes $\left(\frac{m}{2}-p\right)(n-p)$; Now for $i=\alpha$, the pairs for $1 \leq j<\beta$ contributes $\frac{n}{2}-1, j=\beta$ contributes $2 / 3$, $\beta<j \leq n-p$ contributes $\frac{1}{2}\left(\frac{n}{2}-p\right)$ giving the sum $\frac{1}{12}(9 n-6 p-4)$ to $x_{1}$. The vertices lying between $u_{\alpha}$ and $u_{\beta}$ contribute $\frac{1}{2}(n-p)(p-2)$. Consider $u_{\beta}$, it makes pairs with $v_{j}, 1 \leq j \leq \gamma$ and gives $\frac{n}{2}-p+\frac{2}{3}$. Again the vertices on the right of $u_{\beta}$ with the same set give $\left(\frac{m}{2}-p\right)\left[\left(\frac{n}{2}-p\right)+\frac{1}{2}\right]$. Summing all these contributions, we get $B\left(x_{1}, U \mid V\right)$.

(c) Consider the vertex $u_{r}$ for $1 \leq r<\alpha$, now the vertices lying between $u_{r}$ and $u_{\alpha}$ make pairs with all vertices of $V$ giving the contribution $\left(\frac{m}{2}-p-r\right)(n-p)$. Again $u_{\alpha}$ and the vertices lying between $u_{\alpha}$ and $u_{\beta}$ contribute the sum as earlier. But $u_{\beta}$ makes pairs with $v_{j}$ for $1 \leq j \leq \gamma$ and gives $\frac{1}{2}\left(\frac{n}{2}-p\right)+\frac{1}{3}$ and the right of $u_{\beta}$ has no contribution. Summing all the above values gives $B\left(u_{r}, U \mid V\right)$ for $1 \leq r<\alpha$.

(d) Consider the vertex $u_{\alpha}$, now the vertices $u_{i}$ for $\alpha<i \leq \beta$ give the sum $\frac{1}{2}(n-p)(p-2)+\frac{1}{2}\left(\frac{n}{2}-\right.$ $p)+\frac{1}{3}$.

(e) Consider $u_{r}$ for $\alpha<r \leq\left\lceil\frac{m-p}{2}\right\rceil$. Let $r=\alpha+k$ where $1 \leq k \leq\left\lceil\frac{p}{2}\right\rceil-1$. Now $u_{\alpha}$ and $u_{\beta}$ gives $u_{\alpha+k}$ the same contribution as $\frac{1}{2}\left(\frac{n}{2}-p\right)+\frac{1}{3}$. The vertices lying between $u_{\alpha}$ and $u_{\alpha+k}$ gives the sum $(k-1)\left(\frac{n}{2}-p+\frac{1}{2}\right)+\frac{k(k-1)}{2}$ and between $u_{\alpha+k}$ and $u_{\beta}$ gives $\frac{1}{2}(n-k-p)(p-k-2)$. The total of these contribution gives the expression for $B\left(u_{r}, U \mid V\right)$ for $r>\alpha$.

Case 2: Both $C_{m}$ and $C_{n}$ are odd. 
Consider $u_{\alpha}$ and $u_{\beta}$, a pair of extreme vertices of the end vertices $x_{p}$ and $x_{1}$ of $P$ where $\alpha=$ $\frac{m+1}{2}-p+1$ and $\beta=\frac{m-1}{2}$ and $v_{\delta}$ and $v_{\gamma}$ be their eccentric vertices in $C^{\prime}$ where $\delta=\frac{n-1}{2}$, $\gamma=\frac{n+1}{2}-p+1$. If $u_{i} \in U$ and $v_{j} \in V$, then for $x_{r} \in P$ where $1<r<p,\left\{\left(u_{i}, v_{j}\right): 1 \leq i<\alpha\right.$, $\delta<j \leq n-p\}$ or $\left\{\left(u_{i}, v_{j}\right): \beta<i \leq m-p, 1 \leq j<\gamma\right\}$ contributes the sum $\left(\frac{m+1}{2}-p\right)\left(\frac{n+1}{2}-p\right)$. Since there exists no more pair, $B\left(x_{r}, U \mid V\right)=2\left(\frac{m+1}{2}-p\right)\left(\frac{n+1}{2}-p\right)$. Consider the vertex $x_{1}$. Now the pairs $\left\{\left(u_{i}, v_{j}\right): 1 \leq i<\alpha, 1 \leq j \leq n-p\right\}$ contributes the sum $\left(\frac{m+1}{2}-p\right)(n-p)$. The vertices $\left\{u_{i}: \alpha \leq\right.$ $i \leq \beta\}$ contributes the sum $\frac{1}{2}(n-p)(p-1)$ and the pairs $\left\{\left(u_{i}, v_{j}\right): \beta<i \leq m-p, 1 \leq j \leq \frac{n+1}{2}-p\right\}$ contributes the sum $\left(\frac{m+1}{2}-p\right)\left(\frac{n+1}{2}-p\right)$. Hence $B\left(x_{1}, U \mid V\right)=\left(\frac{m+1}{2}-p\right)\left(\frac{n+1}{2}-p\right)+(n-p)\left(\frac{m+p}{2}-p\right)$. Consider the vertex $u_{r}, 1 \leq r<\alpha$, the vertices lying between $u_{r}$ and $u_{\alpha}$ contributes the sum $(\alpha-r-1)(n-p)$, vertices from $u_{\alpha}$ to $u_{\beta}$ as given above and no vertex from the right. Hence $B\left(u_{r}, U \mid V\right)=\left(\frac{m+1}{2}-p-r\right)(n-p)+\frac{1}{2}(n-p)(p-1)$ for $1 \leq r<\alpha$. For $u_{\alpha}$, the vertices from $u_{\alpha+1}$ to $u_{\beta}$ contributes $\frac{1}{2}(p-2)(n-p-1)$. Hence $B\left(u_{\alpha}, U \mid V\right)=\frac{1}{2}(p-2)(n-p-1)$. Consider a vertex on the left of $u_{\alpha}$, say $u_{\alpha+k}$, then no vertex on the right of $u_{\alpha}$ is considered. The vertices from $u_{\alpha}$ to $u_{\alpha+k-1}$ contributes $\frac{1}{2} k(n+k-2 p+1)$ and the vertices from $u_{\alpha+k+1}$ to $u_{\beta}$ contribute $\frac{1}{2}(p-k-2)(n-p-k-1)$ and no more vertex from the right. Hence, $B\left(u_{\alpha+k}, U \mid V\right)=$ $\frac{1}{2} k(n+k-2 p+1)+\frac{1}{2}(p-k-2)(n-p-k-1)$.

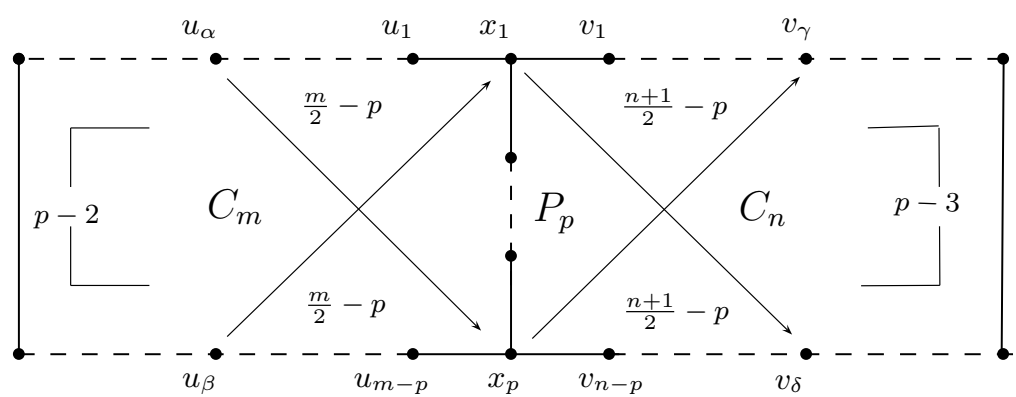

Figure 3. Even and odd cycles merged along a common subgraph

Case 3: $C_{m}$ is even and $C_{n}$ is odd.

Let $u_{\alpha}$ and $u_{\beta}$ where $\alpha=\frac{m}{2}-p+1, \beta=\frac{m}{2}$ be the eccentric vertices in $C_{m}$ for the end vertices $x_{p}, x_{1}$ of $P$ and $v_{\delta}$ and $v_{\gamma}$ be their eccentric vertices in $C^{\prime}$ where $\delta=\frac{n-1}{2}, \gamma=\frac{n+1}{2}-p+1$. See Figure 3.

Consider $x_{r} \in P$ such that $1<r<p$. If $u_{i} \in U$ and $v_{j} \in V$, then for each pair $\left\{\left(u i, v_{j}\right): 1 \leq i<\alpha\right.$, $\delta<j \leq n-p$ or $\beta<i \leq m-p, 1 \leq j<\gamma\}$ there exists a geodesic passing through $x_{r}$ and contributes the sum $\left(\frac{m}{2}-\bar{p}\right)\left(\frac{n+1}{2}-p\right)$ and $\frac{1}{2}\left(\frac{n+1}{2}-p\right)$ for $i=\alpha$. Since there exists no such other pair, $B\left(x_{r}, U \mid V\right)=\frac{1}{2}(m-2 p+1)(n-2 p+1)$.

Consider the vertex $x_{1}$. Now $\left\{\left(u_{i}, v_{j}\right): 1 \leq i<\alpha, 1 \leq j \leq n-p\right\}$ contributes the sum $\left(\frac{m}{2}-p\right)(n-p)$. $\left\{\left(u_{\alpha}, v_{j}\right): 1 \leq j \leq \delta\right\}$ and $\left\{\left(u_{\alpha}, v_{j}\right): \delta<j \leq n-p\right\}$ contributes the sum $\frac{n-1}{2}+\frac{1}{2}\left(\frac{n+1}{2}-p\right)$. $\left\{\left(u_{\beta}, v_{j}\right): 1 \leq j \leq \gamma-1\right\}$ contributes the sum $\frac{n+1}{2}-p$. The vertices lying between $u_{\alpha}$ and $u_{\beta}$ contributes the sum $\frac{1}{2}(n-p)(p-2)$ and each vertex on the right of $u_{\beta}$ gives the sum $\frac{n+1}{2}-p$. The total of these sums gives $B\left(x_{1}, U \mid V\right)$ which is $\frac{1}{4}\left[3 m n-(4 p-1)(m+n)+6 p^{2}-4 p+1\right]$.

Consider the vertex $u_{r}$, for $1 \leq r<\alpha$. Now the vertices lying between $u_{r}$ and $u_{\alpha}$ contributes the sum $(\alpha-r-1)(n-p)$. Again $u_{\alpha}$ and the vertices lying between $u_{\alpha}$ and $u_{\beta}$ have the same contribution as mentioned above, $u_{\beta}$ gives $\frac{1}{2}\left(\frac{n+1}{2}-p\right)$. For the vertex $u_{\alpha}$, vertices from $u_{\alpha+1}$ to $u_{\beta-1}$ and then $u_{\beta}$ contributes to it as earlier. Consider a vertex on the right of $u_{\alpha}$, say $u_{\alpha+k}$. Again $u_{\alpha}$ and $u_{\beta}$ contributes the same as $\frac{1}{2}\left(\frac{n+1}{2}-p\right)$. The vertices lying between $u_{\alpha}$ and $u_{\alpha+k}$ contribute $(k-1)\left(\frac{n+1}{2}-p\right)+\frac{1}{2} k(k-1)$. The vertices lying between $u_{\alpha+k}$ and $u_{\beta}$ contribute $\frac{1}{2}(n-p-k)(p-k-2)$. The sum of these gives $B\left(u_{\alpha+k}, U \mid V\right)$. Consider the vertex $v_{r}$ in $C_{n}$ for 
$1 \leq r<\gamma$. The vertices lying between $v_{r}$ and $v_{\gamma}$ offers $\left(\frac{n+1}{2}-p-r\right)(m-p)$ and the vertices from $v_{\gamma}$ to $v_{\delta}$ offers $(p-1)(m-p) / 2$ giving $B\left(v_{r}, U \mid V\right)=(m-p)(n-p-2 r) / 2$. For $v_{\gamma}$, the vertices from $v_{\gamma+1}$ to $v_{\delta}$ gives $B\left(v_{\gamma}, U \mid V\right)=1 / 2\left[m(p-2)-p^{2}+p+2\right]$. For $v_{\gamma+k}, 1 \leq k \leq p-2$, the vertices from $v_{\gamma+k+1}$ to $v_{\delta}$ offers $\frac{1}{2}(p-k-2)(m-p-k-1)$ and vertices from $v_{\gamma}$ to $v_{\gamma+k-1}$ offers $\frac{k}{2}(m+k-2+1)$ so that vertices symmetric from $v_{\gamma}$ and $v_{\delta}$ offers the same giving $B\left(v_{\gamma+k}, U \mid V\right)=k(k-p+2)+(p / 2)(m-p+1)-m+1$.

Corollary 3.7. Let $G$ be the graph obtained by merging $m$ copies of cycle $C_{n}$ along a common path $P_{p}=\left\{x_{1}, \ldots, x_{p}\right\}$ as common subgraph where $p<\left\lceil\frac{n}{2}\right\rceil$ and $U=\left\{u_{1}, \ldots, u_{n-p}\right\}$ be the vertex sets of $C_{n}-P_{p}$. Then the betweenness centrality of $C_{n}$ in $G$ is given by

$$
\begin{aligned}
& B_{G}\left(x_{r}\right)=m B_{C_{n}}\left(x_{r}\right)-(m-1)(r-1)(p-r)+\left(\begin{array}{c}
m \\
2
\end{array}\right) B\left(x_{r}, U \mid V\right), \text { for } x_{r} \in P_{p}, \\
& B_{G}\left(u_{r}\right)=B_{C_{n}}\left(u_{r}\right)+(m-1) B\left(u_{r}, U \mid V\right), \text { for } u_{r} \in U .
\end{aligned}
$$

where $B\left(x_{r}, U \mid V\right)$ and $B\left(u_{r}, U \mid V\right)$ are given by

Case 1: If $C_{n}$ is even, then

$$
\begin{aligned}
& B\left(x_{r}, U \mid V\right)= \begin{cases}n^{2} / 2-2 n(p-1 / 2)+2\left(p^{2}-p+1 / 3\right), & \text { for } 1<r<p, \\
3 n^{2} / 4-2 n(p-1 / 4)+\left(9 p^{2}-6 p+2\right) / 6, & \text { for } r=1, p .\end{cases} \\
& B\left(u_{r}, U \mid V\right)= \begin{cases}(n-p)(n-p-2 r) / 2, & \text { for } 1 \leq r \leq \frac{n}{2}-p, \\
n(2 p-3) / 4-\left(3 p^{2}-3 p-2\right) / 6, & \text { for } r=\frac{n}{2}-p+1, \\
k(k-p+1)+(n-p)(p-2) / 2+1 / 6, & \text { for } r=\frac{n}{2}-p+1+k, 1 \leq k \leq p-2 .\end{cases}
\end{aligned}
$$

Case 2: If $C_{n}$ is odd, then

$$
\begin{gathered}
B\left(x_{r}, U \mid V\right)= \begin{cases}2[(n+1) / 2-p]^{2}, & \text { for } 1<r<p, \\
3 n^{2} / 4-2 n(p-1 / 4)+\left(6 p^{2}-4 p+1\right) / 4, & \text { for } r=1, p .\end{cases} \\
B\left(u_{r}, U \mid V\right)= \begin{cases}(n-p)(n-p-2 r) / 2, & \text { for } 1 \leq r \leq \frac{n+1}{2}-p, \\
(p-2)(n-p-1) / 2, & \text { for } r=\frac{n+1}{2}-p+1, \\
k(k-p+2)+(p-2)(n-p-1) / 2, & \text { for } r=\frac{n+1}{2}-p+1+k, 1 \leq k \leq p-2 .\end{cases}
\end{gathered}
$$

Proposition 3.8. Let $G$ be the graph obtained by merging both ends of $m$ copies of paths $P_{n}$ together where $V\left(P_{n}\right)=\{1,2, \ldots, n\}$. Then the betweenness centrality of $G$ is given by

$$
B(r)= \begin{cases}\frac{1}{4} m(m-1)(n-2)^{2}, & \text { for } r=1, n, \\ \frac{1}{2}(n-1)(n-3)+\frac{1}{m}+\frac{(m-2)}{2}\left[(n-1-r)^{2}+(r-2)^{2}\right], & \text { for } 1<r<n .\end{cases}
$$

Proof. Since the ends of $m$ copies of path $P_{n}$ are separately merged, any two copies of $P_{n}$ form an even cycle $C_{2 n-2}$ in $G$. Since there are $\left(\begin{array}{c}m \\ 2\end{array}\right)$ such cycles, $B(r)=\left(\begin{array}{c}m \\ 2\end{array}\right) B_{C_{2 n-2}}(r)=\frac{1}{4} m(m-1)(n-2)^{2}$ for $r=1, n$. Consider an internal vertex of any path $P_{n}$ say $P_{n}^{(i)}$. Now $P_{n}^{(i)}$ and $P_{n}^{(j)}$ for $i \neq j$ form a cycle of $2 n-2$ vertices in $G$, where the pair of end vertices gives the centrality $\frac{1}{m}$ instead of $\frac{1}{2}$. Consider the path $P_{n}^{(k)}$ where $k \neq j \neq i$. Let $U_{i}$ and $U_{k}$ denotes the vertex sets of $P_{n-2}^{(i)}$ and $P_{n-2}^{(k)}$ respectively where the merged end vertices 1 and $n$ are deleted. Therefore, for any internal vertex $r$ of $P_{n}$,

$$
\begin{aligned}
B_{G}(r) & =B_{C_{2 n-2}}(r)-\frac{1}{2}+\frac{1}{m}+\sum_{k \neq i \neq j} B\left(r, U_{i} \mid U_{k}\right) \\
& =\frac{1}{2}(n-1)(n-3)+\frac{1}{m}+\frac{(m-2)}{2}\left[(n-1-r)^{2}+(r-2)^{2}\right]
\end{aligned}
$$


since,

$$
\begin{aligned}
B\left(r, U_{i}, \mid U_{k}\right) & =\frac{[n-(r+1)]^{2}}{2}+\frac{1}{2}[1+3+\ldots(r-2) \text { terms }] \\
& =\frac{1}{2}\left[(n-1-r)^{2}+(r-2)^{2}\right] .
\end{aligned}
$$

\subsection{Edge-amalgamation of graphs}

The edge amalgamation of $\left\{G_{i}\right\}_{i=1}^{n}$ is the graph obtained by taking the union of all the $G_{i}$ and identifying their fixed edges. An amalgamation of two edges $e_{1}=u_{1} v_{1}$ of a graph $G_{1}$ and $e_{2}=u_{2} v_{2}$ of a graph $G_{2}$ is a graph created by identifying $u_{1}$ with $u_{2}$ and $v_{1}$ with $v_{2}$ and then deleting one of the two edges corresponding to $e_{1}$ or $e_{2}$. The other edge will be called the amalgamated edge.

The edge amalgamation of cycles are called generalized books [5].

Proposition 3.9. Let two cycles $C_{m}$ and $C_{n}$ are connected by merging a pair of edges. Let $u_{i}, v_{i}$ be any vertices on $C_{m}$ and $C_{n}$ respectively at a distance $i$ from the merged edge. Then betweenness centrality of the resulting graph is given by

Case 1: If both $C_{m}$ and $C_{n}$ are even, then

$$
B\left(u_{i}\right)= \begin{cases}\frac{(m-2)^{2}}{8}+\frac{(n-2)^{2}}{8}+\frac{(m-3)(n-3)}{4}+\frac{(m-2)(n-2)}{2}+\frac{1}{12}, & \text { for } i=0, \\ \frac{(m-2)^{2}}{8}+\frac{(n-2)(m-2-2 i)}{2}, & \text { for } 1 \leq i<\frac{m}{2}-1, \\ \frac{(m-2)^{2}}{8}+\frac{n}{4}-\frac{2}{3}, & \text { for } i=\frac{m}{2}-1 .\end{cases}
$$

$B\left(v_{i}\right)$ are obtained on interchanging $m$ and $n$.

Case 2: If both $C_{m}$ and $C_{n}$ are odd (See Figure 4), then

$$
B\left(u_{i}\right)= \begin{cases}\frac{(m-1)(m-3)}{8}+\frac{(n-1)(n-3)}{8}+\frac{(m-3)(n-3)}{4}+\frac{(m-2)(n-2)}{2}, & \text { for } i=0, \\ \frac{(m-1)(m-3)}{8}+\frac{(n-2)(m-2-2 i)}{2}, & \text { for } 1 \leq i<\frac{m-1}{2} \\ \frac{(m-1)(m-3)}{8}, & \text { for } i=\frac{m-1}{2} .\end{cases}
$$

$B\left(v_{i}\right)$ are obtained on interchanging $m$ and $n$.

Case 3: If $C_{m}$ is even and $C_{n}$ is odd, then

$$
\begin{aligned}
& B\left(u_{i}\right)= \begin{cases}\frac{(m-2)^{2}}{8}+\frac{(n-1)(n-3)}{8}+\frac{(m-3)(n-3)}{4}+\frac{(m-2)(n-2)}{2}, & \text { for } i=0, \\
\frac{(m-2)^{2}}{8}+\frac{(n-2)(m-2-2 i)}{2}, & \text { for } 1 \leq i<\frac{m}{2}-1, \\
\frac{(m-2)^{2}}{8}+\frac{n}{4}-\frac{2}{3}, & \text { for } i=\frac{m}{2}-1 .\end{cases} \\
& B\left(v_{i}\right)= \begin{cases}\frac{(n-1)(n-3)}{8}+(m-2)(n-2-2 i) / 2, & \text { for } 1 \leq i<\frac{n-1}{2}, \\
\frac{(n-1)(n-3)}{8}, & \text { for } i=\frac{n-1}{2} .\end{cases}
\end{aligned}
$$




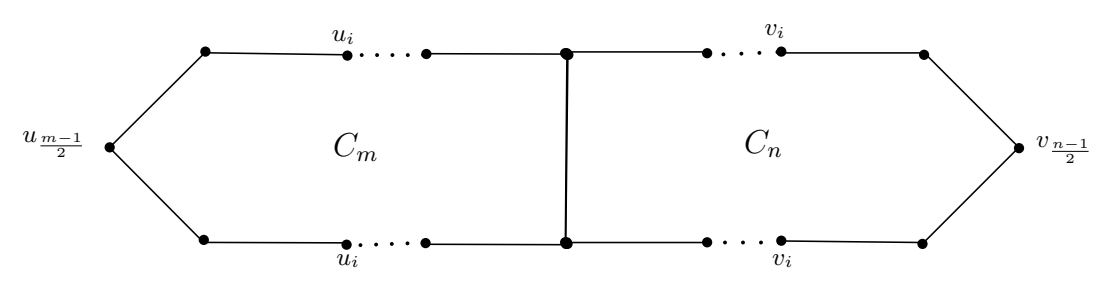

Figure 4. Two odd cycles $C_{m}$ and $C_{n}$ identifying a pair of edges

Proof. Consider a merged vertex. Since the merged vertex lies on both cycles $C_{m}$ and $C_{n}$, each cycle induces a value for its betweenness centrality. $C_{m}$ against $C_{n}$ also induces a value. The betweenness centrality of merged vertex is their sum. For any other vertex $u$, it lies on one of the cycle and betweenness centrality is the sum of the betweenness centrality induced by that cycle and the other one on it.

Corollary 3.10. Let the odd cycles $C_{n_{1}}, \ldots, C_{n_{m}}$ share a common edge. Then, for $u_{0}$, an end vertex of the common edge and $u_{i}$, a vertex in $C_{n_{k}}$ at a distance $i$ from the common edge, we have

$$
\begin{gathered}
B\left(u_{0}\right)=\frac{1}{8} \sum_{i}\left(n_{i}-1\right)\left(n_{i}-3\right)+\frac{1}{4} \sum_{i<j}\left(n_{i}-3\right)\left(n_{j}-3\right)+\frac{1}{2} \sum_{i<j}\left(n_{i}-2\right)\left(n_{j}-2\right), \\
B\left(u_{i}\right)= \begin{cases}\frac{\left(n_{k}-1\right)\left(n_{k}-3\right)}{8}+\left(\frac{n_{k}}{2}-1-i\right) \sum_{i \neq k}\left(n_{i}-2\right), & \text { for } 1 \leq i<\frac{n_{k}-1}{2}, \\
\frac{\left(n_{k}-1\right)\left(n_{k}-3\right)}{8}, & \text { for } i=\frac{n_{k}-1}{2} .\end{cases}
\end{gathered}
$$

Corollary 3.11. If $m$ copies of $n$-cycles, each are merged at an edge, then for $u_{0}$, an end vertex of the common edge and $u_{i}$, a vertex at a distance $i$ from the common edge in any $n$-cycle (See Figure 5), then we have

Case 1: If $n$ is even

$$
B\left(u_{i}\right)= \begin{cases}\frac{m(n-2)^{2}}{8}+\left(\begin{array}{c}
m \\
2
\end{array}\right)\left[\frac{(n-2)^{2}}{2}+\frac{(n-3)^{2}}{4}+\frac{1}{12}\right], & \text { when } i=0, \\
\frac{(n-2)^{2}}{8}+(m-1)(n-2)\left(\frac{n}{2}-1-i\right), & \text { when } 1 \leq i<\frac{n}{2}-1, \\
\frac{(n-2)^{2}}{8}+(m-1)\left(\frac{n}{4}-\frac{2}{3}\right), & \text { when } i=\frac{n}{2}-1 .\end{cases}
$$

Case 2: If $n$ is odd

$$
B\left(u_{i}\right)= \begin{cases}\frac{m(n-1)(n-3)}{8}+\left(\begin{array}{c}
m \\
2
\end{array}\right)\left[\frac{(n-2)^{2}}{2}+\frac{(n-3)^{2}}{4}\right], & \text { when } i=0 \\
\frac{(n-1)(n-3)}{8}+(m-1)(n-2)\left(\frac{n}{2}-1-i\right), & \text { when } 1 \leq i<\frac{n-1}{2} \\
\frac{(n-1)(n-3)}{8}, & \text { when } i=\frac{n-1}{2}\end{cases}
$$




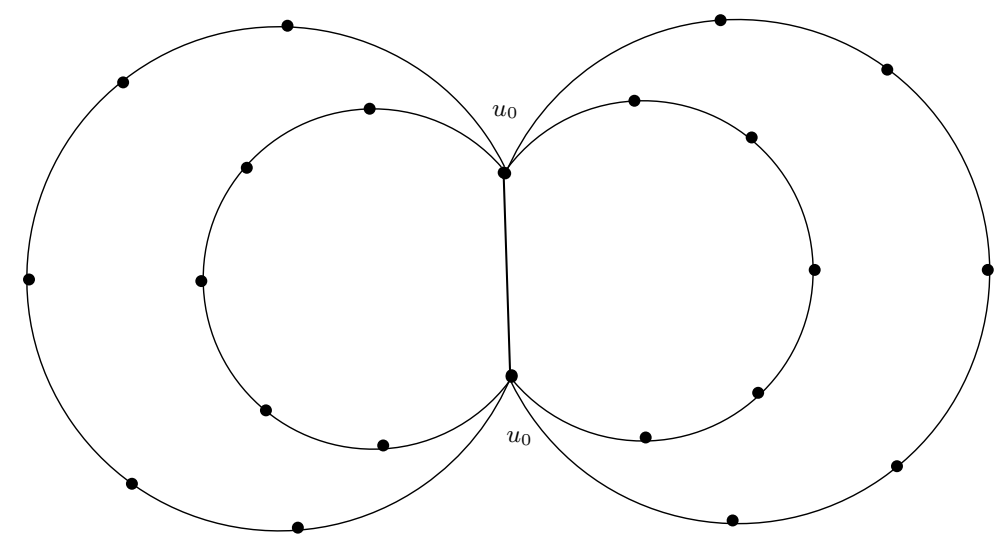

Figure 5. Four 7-cycles sharing a common edge

\subsection{Vertex-amalgamation of graphs}

Definition 3.12. [6] A graph $G$ in which a vertex is distinguished from other vertices is called a rooted graph and the vertex is called a root (or terminal) of $G$.

Identifying the terminal vertices of two graphs is known as vertex-amalgamation and the new graph obtained by vertex-amalgamation of $G_{1}$ and $G_{2}$ is denoted by $G_{1} \cdot G_{2}$ [4].

Proposition 3.13. Let $G_{1}, \ldots, G_{k}$ be vertex disjoint graphs of order $n_{1}, \ldots, n_{k}$ respectively and $G$, the graph obtained by identifying the vertices $v_{i} \in G_{i}, \forall i$; if $v_{0}$ denotes the merged vertex and $v\left(\neq v_{i}\right) \in G_{i}$, then

$$
\begin{gathered}
B_{G}\left(v_{0}\right)=\sum_{i=1}^{k} B_{G_{i}}\left(v_{i}\right)+\sum_{i<j}\left(n_{i}-1\right)\left(n_{j}-1\right) \text { and } \\
B_{G}(v)=B_{G_{i}}(v)+B_{G_{i}}\left(v, v_{0}\right) \sum_{j \neq i}\left(n_{j}-1\right) .
\end{gathered}
$$

Proof. Consider the graph $G$ obtained by identifying the vertices $v_{i} \in G_{i}$. If $v_{0}$ denotes the merged vertex, i.e, $v_{i}=v_{0}$ for all $i, v_{0}$ is a cut vertex (See Figure 6 ) and the removal of $v_{0}$ disconnects the graph $G$ into $k$ components. The betweenness centrality of $v_{0}$ in $G$ is calculated over all pairs of vertices and each pair belongs to the same component or different ones. The pairs of vertices lying in the same component give the sum $\sum_{i=1}^{k} B_{G_{i}}\left(v_{i}\right)$ and the pairs of vertices lying in different components give $\sum_{i<j}\left(n_{i}-1\right)\left(n_{j}-1\right)$. Now their sum gives the result. For $v \in G_{i}, v \neq v_{i}$, a pair of vertices in $G$ provides a contribution to the centrality of $v$, if one of which belongs to $G_{i}$. If $B_{G_{i}}\left(v, v_{0}\right)$ denotes the betweenness centrality of $v$ induced by $v_{0}$ in $G_{i}$, then clearly $B_{G}(v)=B_{G_{i}}\left(v_{i}\right)+B_{G_{i}}\left(v, v_{0}\right) \sum_{j \neq i}\left(n_{j}-1\right)$.

Algorithm 3.14. Algorithm for Betweenness Computation of Merged Vertex $v_{0}$ in a Vertex Amalgamation

Require: Vertex disjoint graphs $G_{1}, G_{2}, \ldots, G_{k}$ of order $n_{1}, n_{2}, \ldots, n_{k}$, and $v_{0}$ the merged vertex

Ensure: Betweenness of vertex $v_{0}, B_{G}\left(v_{0}\right)$

1: Compute betweenness of $v_{i}$ in $G_{i}, B_{G_{i}}\left(v_{i}\right)$ for $i=1, \ldots, k$. Add all these betweenness values to get B ComponentSum

2: Find $B_{\text {Contribution }}=\sum_{i=1, \ldots, k-1}\left(n_{i}-1\right) *\left(n_{j}-1\right)$ for $i<j$.

3: Determine $B_{G}\left(v_{0}\right)=B_{\text {ComponentSum }}+B_{\text {Contribution }}$ 


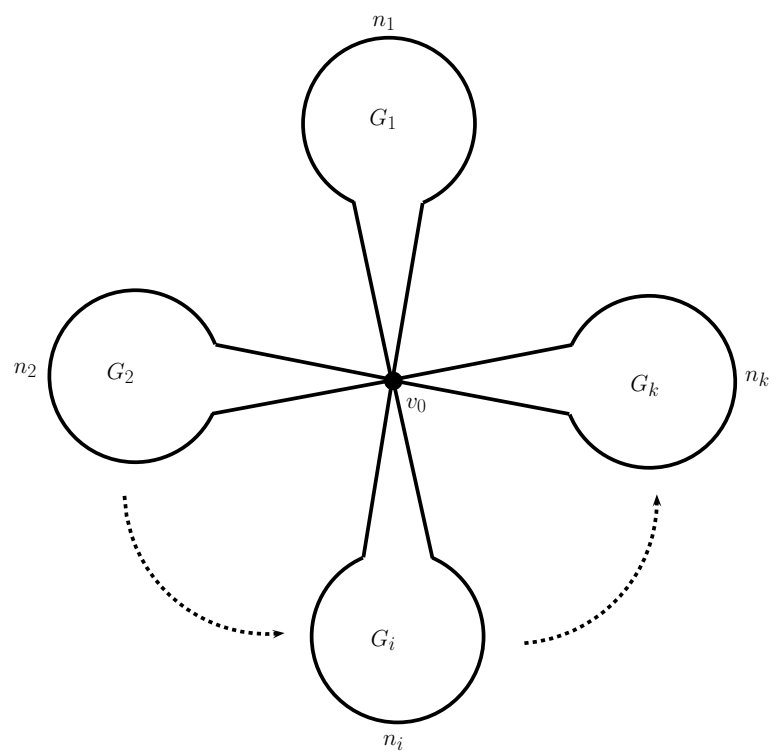

Figure 6. Vertex amalgamation of $G_{i}$ 's

Theorem 3.15. Let $G$ be the graph obtained by merging graphs $\left\{G_{i}\right\}_{i=1}^{k}$, on a common root vertex $v_{0}$. Then Algorithm 3.14 correctly computes the betweenness value of merged vertex $v_{0}$ in $O\left(n^{\prime} m^{\prime}\right)$ times where $n^{\prime}$ is the order of the largest component graph $G_{i}$ and $m^{\prime}$ the number of edges in $G_{i}$.

Proof. Proof of Algorithm 3.14 follows from Proposition 3.13.

Algorithm 3.16. Algorithm for Betweenness Computation of Non-terminal Vertex $v$ in $G_{i}$ in a Vertex Amalgamation

Require: Vertex disjoint graphs $G_{1}, G_{2}, \ldots, G_{k}$ of order $n_{1}, n_{2}, \ldots, n_{k}, v_{0}$ the merged vertex, vertex $v$ a non-terminal vertex in $G_{i}$.

Ensure: Betweenness of vertex $v, B_{G}(v)$

1: Compute betweenness of $v$ in $G_{i}, B_{G_{i}}(v)$.

2: Compute betweenness of $v$ induced by $v_{0}$ in $G_{i} B_{G_{i}}\left(v, v_{0}\right)$.

3: Find $B_{\text {Contribution }}=\sum_{j=1, \ldots, k ; j \neq i}\left(n_{j}-1\right)$.

4: Determine $B_{G}(v)=B_{G_{i}}(v)+B_{G_{i}}\left(v, v_{0}\right) * B_{\text {Contribution }}$

Theorem 3.17. Let $G$ be the graph obtained by merging graphs $\left\{G_{i}\right\}_{i=1}^{k}$ on a common root vertex $v_{0}$. Then Algorithm 3.16 correctly computes the betweenness value of non-terminal vertex $v$ in $O\left(n_{i} m_{i}\right)$ times where $n_{i}$ is the order of the component graph $G_{i}$ and $m_{i}$ the number of edges in $G_{i}$.

Proof. Proof of Algorithm 3.16 follows from Proposition 3.13.

Proposition 3.18. Let $G_{1}, \ldots, G_{k}$ be vertex disjoint graphs of order $n_{1}, \ldots, n_{k}$. Consider the graph $G$ obtained on joining the vertices $v_{i} \in G_{i}$ to a single vertex $v_{0}$ by means of edges and $v\left(\neq v_{i}\right) \in G_{i}$, then

$$
\begin{aligned}
B_{G}\left(v_{0}\right) & =\sum_{i<j} n_{i} n_{j}, \\
B_{G}\left(v_{i}\right) & =B_{G_{i}}\left(v_{i}\right)+\left(n_{i}-1\right)\left(\sum_{j \neq i} n_{j}+1\right) \text { and } \\
B_{G}(v) & =B_{G_{i}}(v)+B_{G_{i}}\left(v, v_{0}\right) \sum_{j \neq i}\left(n_{j}-1\right) .
\end{aligned}
$$


The above result is significant in view of the fact that networks are often connected by joining to a common node.

If $G$ is a rooted graph, the graph $G^{(n)}$ obtained on identifying the root of $n$-copies of $G$ is called a one-point union of $n$ copies of $G$ [9]. We consider the following examples.

\section{Windmill graph}

The windmill graph $K_{n}^{(m)}$ is the one - point union of $m$ copies of the complete graph $K_{n} ; K_{n}^{(m)}$ has $(n-1) m+1$ vertices and $m\left(\begin{array}{c}n \\ 2\end{array}\right)$ edges. For example, $K_{5}^{(4)}$ is given in Figure 7.

Theorem 3.19. The betweenness centrality of windmill graph $K_{n}^{(m)}$ is given by

$$
B(v)= \begin{cases}(n-1)^{2}\left(\begin{array}{c}
m \\
2
\end{array}\right), & \text { for central vertex } \\
0, & \text { for any other vertex }\end{cases}
$$

Proof. The central vertex of $K_{n}^{(m)}$ is a cut vertex and the removal of which disconnects into $m$ components of $K_{n-1}$. Therefore, by Proposition 3.13, the betweenness centrality of the central vertex is given by $\mathcal{C}(n-1, n-1, \ldots m$ times $)=(n-1)^{2}\left(\begin{array}{c}m \\ 2\end{array}\right)$ where $\mathcal{C}\left(n_{1}, n_{2}, \ldots, n_{k}\right)=\sum_{i<j} n_{i} n_{j}$. Since other vertices are vertices of the induced subgraph $K_{n-1}$, their betweenness centrality is zero.

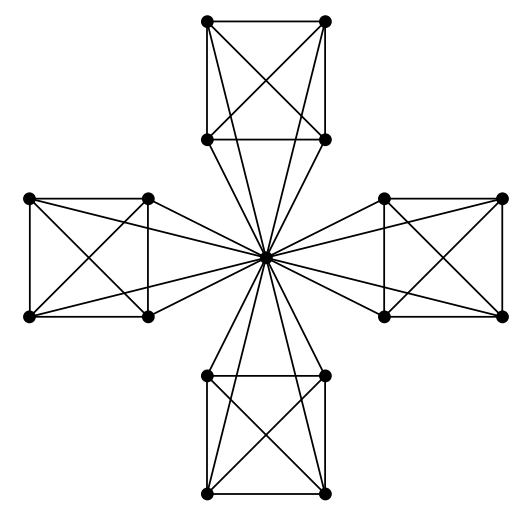

Figure 7. Windmill graph $K_{5}^{(4)}$

The graph $K_{3}^{(t)}$ is called a friendship graph or Dutch $t$-windmill graph. For example, $K_{3}^{(5)}$ is given in Figure 8. Every pair of vertices in $K_{3}^{(t)}$ has exactly one common neighbour. $K_{3}^{(2)}$ is the butterfly graph and $K_{2}^{(n)}$ is the star $S_{1, n}$.

Corollary 3.20. The betweenness centrality of friendship graph $K_{3}^{(n)}$ is given by

$$
B(v)= \begin{cases}4\left(\begin{array}{l}
n \\
2
\end{array}\right), & \text { for central vertex } \\
0, & \text { for any other vertex }\end{cases}
$$

\section{Vertex-amalgamation of cycles}

Now let us consider the case when different cycles merged at a vertex. See Figure 9. 


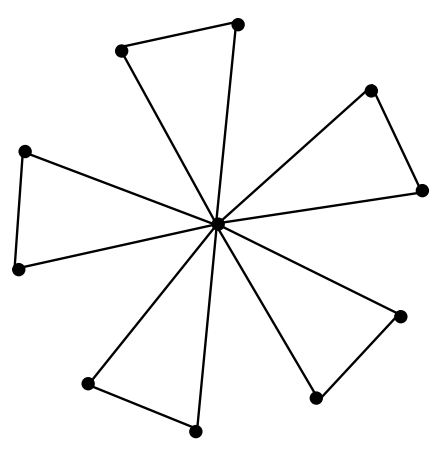

Figure 8. Friendship graph $K_{3}^{(5)}$

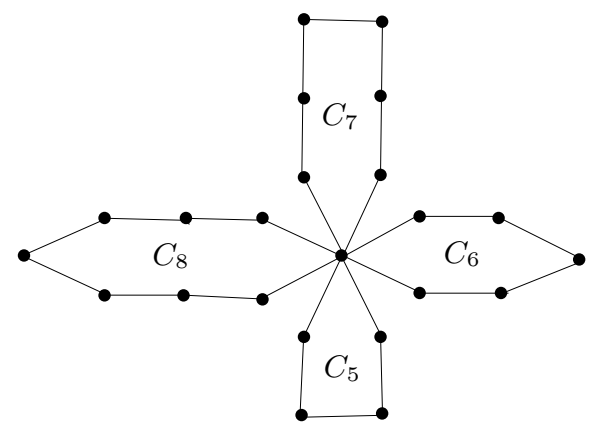

Figure 9. One point union of different cycles

Proposition 3.21. Let the graph $G$ be the union of $m_{1}$ copies of odd cycle $C_{n_{1}}$ and $m_{2}$ copies of even cycle $C_{n_{2}}$ with a common vertex $v_{0}$. In any cycle $C$, let $v_{i}$ denotes the vertex such that $d\left(v_{i}, v_{0}\right)=i$, then,

$$
\begin{aligned}
& B\left(v_{0}\right)=\frac{m_{1}\left(n_{1}-1\right)\left(n_{1}-3\right)}{8}+\frac{m_{2}\left(n_{2}-2\right)^{2}}{8}+\left(n_{1}-1\right)^{2}\left(\begin{array}{c}
m_{1} \\
2
\end{array}\right)+\left(n_{2}-1\right)^{2}\left(\begin{array}{c}
m_{2} \\
2
\end{array}\right) \\
& +\left(n_{1}-1\right)\left(n_{2}-1\right) m_{1} m_{2} \text {, and } \\
& B\left(v_{i}\right)= \begin{cases}\frac{\left(n_{1}-1\right)\left(n_{1}-3\right)}{8}+\left(\frac{n_{1}-1-2 i}{2}\right)\left[\left(m_{1}-1\right)\left(n_{1}-1\right)+m_{2}\left(n_{2}-1\right)\right], & \text { for } v_{i} \in C_{n_{1}}, 1 \leq i \leq \frac{n_{1}-1}{2}, \\
\frac{\left(n_{2}-2\right)^{2}}{8}+\left(\frac{n_{2}-1-2 i}{2}\right)\left[m_{1}\left(n_{1}-1\right)+\left(m_{2}-1\right)\left(n_{2}-1\right)\right], & \text { for } v_{i} \in C_{n_{2}}, 1 \leq i<\frac{n_{2}}{2}, \\
\frac{\left(n_{2}-2\right)^{2}}{8}, & \text { for } v_{i} \in C_{n_{2}}, i=\frac{n_{2}}{2} .\end{cases}
\end{aligned}
$$

Proof. Since $m_{1}$ copies of odd cycles $C_{n_{1}}$ and $m_{2}$ copies of even cycles $C_{n_{2}}$ have a common vertex $v_{0}, v_{0}$ is a cut vertex of $G$ and each cycle contributes its own betweenness centrality to $v_{0}$. Again $v_{0}$ lies on the path joining vertices of different cycles. Each pair of cycles contributes a betweenness centrality $\left(n_{i}-1\right)^{2}$ and there are $\left(\begin{array}{c}m_{i} \\ 2\end{array}\right)$ pairs of such cycles giving $\left(n_{i}-1\right)^{2}\left(\begin{array}{c}m_{i} \\ 2\end{array}\right)$ to the centrality. Considering the three possible combinations odd-odd, even-even and odd-even pairs of cycles, we get the centrality of $v_{0}$ as their sum. Let $v_{i}$ denotes the vertices at a distance $i$ from $v_{0}$. If $v_{i} \in C_{n_{1}}$, an odd cycle, for $i \leq \frac{n_{1}-1}{2}$ then from each vertex lying between $v_{i}$ and $v_{\frac{n_{1}-1}{2}}$, there is a path passing through $v_{i}$ to each vertex of the remaining cycles and consequently there is an increase of $\left(\frac{n_{1}-1-2 i}{2}\right)\left[\left(m_{1}-1\right)\left(n_{1}-1\right)+m_{2}\left(n_{2}-1\right)\right]$ for $B\left(v_{i}\right)$. If $v_{i} \in C_{n_{2}}$, an even cycle, then from each vertex lying between $v_{i}$ and $v_{\frac{n}{2}}$, there is a path passing through 


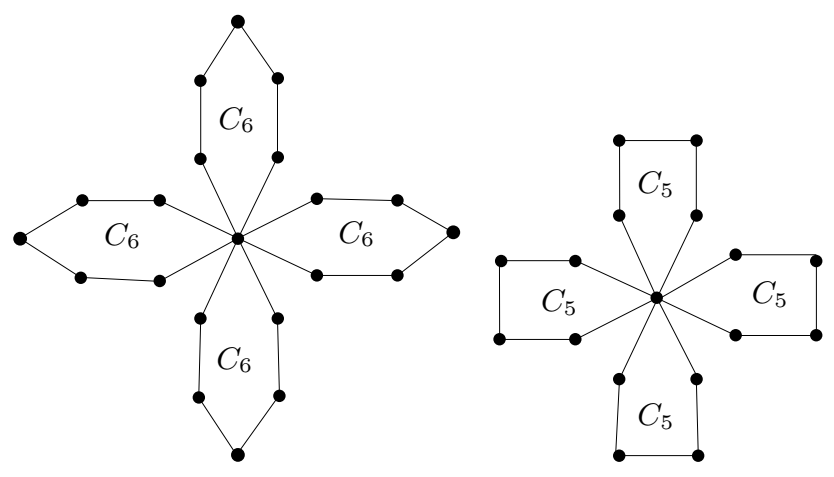

Figure 10. $C_{6}^{(4)}$ and $C_{5}^{(4)}$

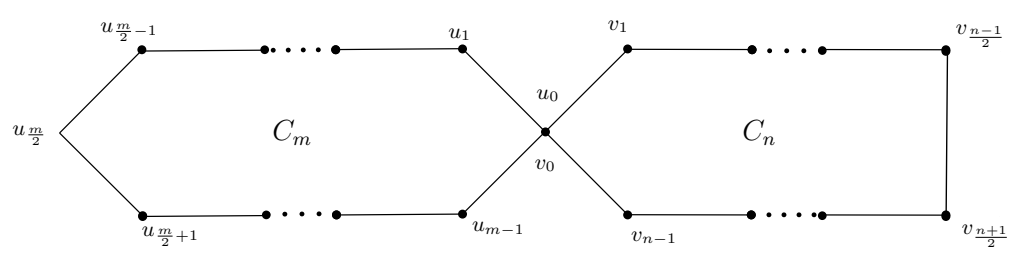

Figure 11. An even cycle $C_{m}$ and an odd cycle $C_{n}$ merged at a vertex

$v_{i}$ to each vertex of the remaining cycles and from the extreme vertex $v_{\frac{n}{2}}$ there are two paths and one of which passes through $v_{i}$. Hence there is an increase of centrality $\left(\frac{n_{2}-1-2 i}{2}\right)\left[m_{1}\left(n_{1}-1\right)+\left(m_{2}-1\right)\left(n_{2}-1\right)\right]$ for $B\left(v_{i}\right)$. Since there is no path passing through the extreme vertex $v_{\frac{n_{2}}{2}}$, its centrality remains the same as that of $C_{n_{2}}$, that is, $\frac{\left(n_{2}-2\right)^{2}}{8}$.

Corollary 3.22. Let $C_{n}^{(m)}$ be the one point union of $m$ copies of $C_{n}$. See Figure 10. If $v_{0}$ is the common vertex and $v_{i} \in C_{n}^{(m)}$ such that $d\left(v_{i}, v_{0}\right)=i$, then the betweenness centrality of $C_{n}^{(m)}$ is given by

Case 1: When $C_{n}$ is even

$$
B\left(v_{i}\right)= \begin{cases}\frac{m(n-2)^{2}}{8}+\left(\begin{array}{c}
m \\
2
\end{array}\right)(n-1)^{2}, & \text { when } i=0, \\
\frac{(n-2)^{2}}{8}+\frac{(m-1)(n-1)(n-1-2 i)}{2}, & \text { when } 1 \leq i<\frac{n}{2}, \\
\frac{(n-2)^{2}}{8}, & \text { when } i=\frac{n}{2} .\end{cases}
$$

Case 2: When $C_{n}$ is odd

$$
B\left(v_{i}\right)= \begin{cases}\frac{m(n-1)(n-3)}{8}+\left(\begin{array}{c}
m \\
2
\end{array}\right)(n-1)^{2}, & \text { when } i=0, \\
\frac{(n-1)(n-3)}{8}+\frac{(m-1)(n-1)(n-1-2 i)}{2}, & \text { when } 1 \leq i \leq \frac{n-1}{2} .\end{cases}
$$

Corollary 3.23. Let $G$ be the graph obtained by merging the vertices $u_{0} \in C_{m}$ and $v_{0} \in C_{n}$ and let $u_{i} \in C_{m}$ and $v_{j} \in C_{n}$ such that $d\left(u_{i}, u_{0}\right)=i$ and $d\left(v_{j}, v_{0}\right)=j$. Then the betweenness centrality of $G$ is given by 
Case 1: If both $C_{m}$ and $C_{n}$ are even, then

$$
B\left(u_{i}\right)= \begin{cases}\frac{(m-2)^{2}}{8}+\frac{(n-2)^{2}}{8}+(m-1)(n-1), & \text { when } i=0, \\ \frac{(m-2)^{2}}{8}+\frac{(n-1)(m-1-2 i)}{2}, & \text { when } 1 \leq i<\frac{m}{2}, \\ \frac{(m-2)^{2}}{8}, & \text { when } i=\frac{m}{2} .\end{cases}
$$

$B\left(v_{i}\right)$ are obtained by interchanging $m$ and $n$.

Case 2: If both $C_{m}$ and $C_{n}$ are odd, then

$$
B\left(u_{i}\right)= \begin{cases}\frac{(m-1)(m-3)}{8}+\frac{(n-1)(n-3)}{8}+(m-1)(n-1), & \text { when } i=0, \\ \frac{(m-1)(m-3)}{8}+\frac{(n-1)(m-1-2 i)}{2}, & \text { when } 1 \leq i \leq \frac{m-1}{2} .\end{cases}
$$

$B\left(v_{i}\right)$ are obtained by interchanging $m$ and $n$.

Case 3: If $C_{m}$ is even and $C_{n}$ is odd (see Figure 11), then

$$
\begin{aligned}
& B\left(u_{i}\right)= \begin{cases}\frac{(m-2)^{2}}{8}+\frac{(n-1)(n-3)}{8}+(m-1)(n-1), & \text { when } i=0 \\
\frac{(m-2)^{2}}{8}+\frac{(n-1)(m-1-2 i)}{2}, & \text { when } 1 \leq i<\frac{m}{2} \\
\frac{(m-2)^{2}}{8}, & \text { when } i=\frac{m}{2}\end{cases} \\
& B\left(v_{j}\right)=\frac{(n-1)(n-3)}{8}+\frac{(m-1)(n-1-2 j)}{2}, \text { when } 1 \leq j \leq \frac{n-1}{2}
\end{aligned}
$$

Note: If the two cycles are identical, then

Case 1: $m=n$ an even number.

$$
\begin{aligned}
& B\left(u_{i}\right)=B\left(v_{i}\right), \text { where } \\
& B\left(u_{i}\right)= \begin{cases}\frac{(m-2)^{2}}{4}+(m-1)^{2}, & \text { when } i=0, \\
\frac{(m-2)^{2}}{8}+\frac{(m-1)(m-1-2 i)}{2}, & \text { when } 1 \leq i<\frac{m}{2}, \\
\frac{(m-2)^{2}}{8}, & \text { when } i=\frac{m}{2}\end{cases}
\end{aligned}
$$

Case 2: $m=n=$ an odd number.

$$
\begin{aligned}
& B\left(u_{i}\right)=B\left(v_{i}\right), \text { where } \\
& B\left(u_{i}\right)= \begin{cases}\frac{(m-1)(m-3)}{4}+(m-1)^{2}, & \text { when } i=0, \\
\frac{(m-1)(m-3)}{8}+\frac{(m-1)(m-1-2 i)}{2}, & \text { when } 1 \leq i \leq \frac{m-1}{2} .\end{cases}
\end{aligned}
$$

\section{Conclusion}

Betweenness centrality is a useful metric for analysing graph-structures and networks. When compared to other centrality measures, computation of betweenness centrality is rather difficult as it involves finding the shortest paths between pairs of vertices in a graph. Therefore studying the structure based on graph operations becomes important. Here new graph classes originated by subgraph amalgamation have been studied. This study can be extended to other structures and is therefore helpful for analysing larger classes of graphs. 


\section{References}

[1] A. Bavelas, A mathematical model for group structures, Human Organization 7, Appl. Anthropol. $7(3)$ (1948) 16-30.

[2] U. Brandes, A faster algorithm for betweenness centrality, J. Math. Sociol. 25(2) (2001) 163-177.

[3] L. C. Freeman, A set of measures of centrality based on betweenness, Sociometry 40(1) (1977) 35-41.

[4] R. Frucht, F. Haray, On the corona of two graphs, Aequationes Math. 4(3) (1970) 322-325.

[5] J. A. Gallian, A dynamic survey of graph labeling, Electron. J. Combin. (2009) 1-219.

[6] F. Harary, The number of linear, directed, rooted, and connected graphs, Trans. Amer. Math. Soc. $78(2)$ (1955) 445-463.

[7] S. Kumar, K. Balakrishnan, M. Jathavedan, Betweenness centrality in some classes of graphs, Int. J. Comb. 2014 (2014) 1-12.

[8] S. Kumar, K. Balakrishnan, On the number of geodesics of Petersen graph $G P(n, 2)$, Electronic Notes in Discrete Mathematics 63 (2017) 295-302.

[9] S.-C. Shee, Y.-S. Ho, The cordiality of one-point union of $n$ copies of a graph, Discrete Math. 117(1-3) (1993) 225-243. 